\title{
Neuromarketing: The New Science of Consumer Behavior
}

\author{
Christophe Morin
}

Published online: 14 January 2011

(C) Springer Science+Business Media, LLC 2011

\begin{abstract}
Neuromarketing is an emerging field that bridges the study of consumer behavior with neuroscience. Controversial when it first emerged in 2002, the field is gaining rapid credibility and adoption among advertising and marketing professionals. Each year, over 400 billion dollars is invested in advertising campaigns. Yet, conventional methods for testing and predicting the effectiveness of those investments have generally failed because they depend on consumers' willingness and competency to describe how they feel when they are exposed to an advertisement. Neuromarketing offers cutting edge methods for directly probing minds without requiring demanding cognitive or conscious participation. This paper discusses the promise of the burgeoning field of neuromarketing and suggests it has the potential to significantly improve the effectiveness of both commercial and cause-related advertising messages around the world.
\end{abstract}

Keywords Neuromarketing $\cdot$ Advertising $\cdot$ Marketing research $\cdot$ Consumer behavior $\cdot f$ MRI $\cdot E E G \cdot$ Neuroscience

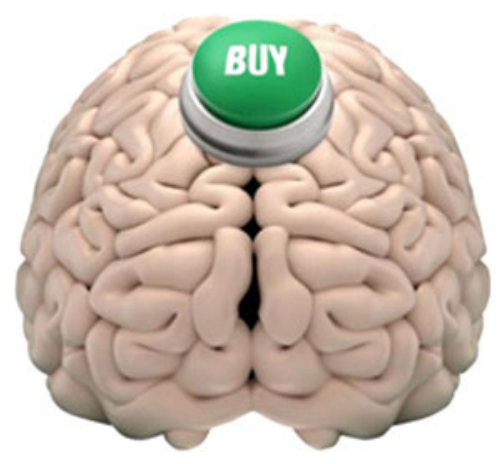

Imagine John, a healthy middle-aged man entering a room filled with somber people dressed in white lab coats.

C. Morin $(\bowtie)$

110 Sir Francis Drake Blvd,

San Anselmo, CA 94960, USA

e-mail: christophe@salesbrain.com
John is worried. Maybe this is a mistake, he thinks. But already one of the earnest technicians whose smile seems just a little too forced is shaking his hand and spurring him forward. He ensures him everything will go according to plan. "It takes about 30 minutes to do the scan" he says "and as long as you don't pay attention to the noise, you will be fine in the tunnel". John does not feel nearly as relaxed now as he was when he agreed to do this experiment. What if the magnetic field in which I agreed to put my entire body kills some of my vital cells? In an instant, he goes from being worried to being terrified. What if the radiation alters my mental abilities? Reluctantly, John lies down on the table and tries to ignore the grinding sound of the pulley propelling him to the center of the tunnel. In a matter of seconds, the machine begins to bombard his head with subatomic particles. "Too late now", he mumbles.

So, who is John? Is he a patient coming for a clinical evaluation or a fresh recruit taking part in a neuromarketing study? John is a consumer, like you. But today, he agreed to be part of a new breed of studies involving the use of the latest tool available to investigate the workings of your mind: an fMRI scanner. The brain has been long described as the most complex structure in the universe. Many consider $f$ MRI the best technological innovation ever developed to conduct clinical and experimental research on the brain. No wonder there has been such tremendous enthusiasm for neuroimaging technology since its emergence in the mid-1980s. Additionally, the rapid progress in mapping the brain's circuitry has fueled the growth of vibrant fields of study such as neuropsychology (understanding psyche through the study of cognitive processes), neurophysiology (understanding the function of our nervous system), neuroethology (understanding animal behavior through the comparative study of our nervous systems), and neuroanatomy (understanding the neural structures of our nervous system).

Clearly, it was only a matter of time before marketers and advertisers would also start considering the possibilities 
of probing consumers' brains using the same equipment favored by neurologists and scientists around the world. Could neuroscience be the holy grail of the study of consumer behavior? Can neuromarketing succeed in developing predictive models that can explain why we buy anything? These are questions that make some people smile, and others cringe.

\section{The (Short) History of Neuromarketing}

The combination of neuro and marketing implies the merging of two fields of study (neuroscience and marketing). The term neuromarketing cannot be attributed to a particular individual as it started appearing somewhat organically around 2002. At the time, a few U.S. companies like Brighthouse and SalesBrain became the first to offer neuromarketing research and consulting services advocating the use of technology and knowledge coming from the field of cognitive neuroscience. Basically, neuromarketing is to marketing what neuropsychology is to psychology. While neuropsychology studies the relationship between the brain and human cognitive and psychological functions, neuromarketing promotes the value of looking at consumer behavior from a brain perspective.

The first scholarly piece of neuromarketing research was performed by Read Montague, Professor of Neuroscience at Baylor College of Medicine in 2003 and published in Neuron in 2004. The study asked a group of people to drink either Pepsi or Coca Cola while their brains were scanned in an fMRI machine. While the conclusions of the study were intriguing, Dr Montague failed to provide a rationale for how our brain handles brand choices. Nevertheless, the study did reveal that different parts of the brain light up if people are aware or not aware of the brand they consume. Specifically, the study suggested that a strong brand such as Coca Cola has the power to "own" a piece of our frontal cortex. The frontal lobe is considered the seat of our executive function (EF) which manages our attention, controls our short-term memory, and does the best of our thinking - especially planning. So according to the study, when people know they are drinking Coca Cola, they actually say they prefer the Coke brand over Pepsi and their EF lights up. However, when they don't know which brand they are consuming, they report that they prefer Pepsi instead. In this latter event, the part of the brain which is most active is not the EF but an older structure nestled in the limbic system. This brain area is responsible for our emotional and instinctual behavior. The Coke and Pepsi study may have not been enough to convince many marketing researchers that neuroscience could help crack the neural code of our decisions, but it was certainly enough to worry many about its potential power.
Indeed, this study triggered a wave of heavy criticism towards neuromarketing because of the fear that it harbored a hidden code to tweak our perceptions below the level of our consciousness. The journal Nature Neuroscience published an article in 2004 entitled "Brain Scam" raising the question of ethics behind neuromarketing studies. Morality of neuromarketers was strongly questioned in the paper. In response, Dr. Michael Brammer, the CEO of Neurosense, a company who was mentioned in the article, eloquently replied to the editor of the journal stating:

I would agree .. in urging caution in the exploitation of any new technology. Scientific rigor and ethical considerations are of paramount importance, but these questions are not confined to commercial activities but rather must apply to all our activities as scientists. Only time will tell whether neuromarketing using $f$ MRI will become an established tool. If our crime is to investigate its value in understanding behavior, and to be paid in the process, we plead guilty.

Notably, this short-lived attack from the media did not dissuade Harper Collins from adding the word "neuromarketing" to its dictionary in 2005. And by 2006, neither the critical article from Nature Neuroscience nor the efforts deployed by the consumer advocacy group Commercial Alert succeeded in curbing the popularity and growth of neuromarketing. Let's explore why.

For too long, both marketers and advertisers have relied on ancient ways to create and assess effective advertising campaigns. Millions of dollars are poured each year into developing products that will never see the light of day. Countless campaigns fail to attract consumer attention and successfully impact our memory banks. Ignoring neuroimaging as a way to understand consumer behavior would be as absurd as astronomers refusing to use electronic telescopes. Placing legitimate worries on ethics aside, there is no question that neuroimaging provides powerful lenses through which we can observe and understand the mind of a consumer.

\section{Understanding the Consumer's Brain}

For decades, marketing research methods have aimed to explain and predict the effectiveness of advertising campaigns. For the most part, however, conventional techniques have failed miserably. Since emotions are strong mediators of how consumers process messages, understanding and modeling cognitive responses to selling messages has always been a methodological challenge. For instance, researchers have primarily relied on consumers' abilities to report how they feel about a particular piece of advertising, either in a confidential setting such a face-to- 
face interview, a survey, or in a group setting such as a focus group. Unfortunately, these methods have considerable limitations. First, they assume that people are actually able to describe their own cognitive process which we now know has many subconscious components. Second, numerous factors motivate research participants to distort the reporting of their feelings, including incentives, time constraints, or peer pressure.

In this challenging context, the emergence of neuroimaging techniques has offered exciting methodological alternatives. Such techniques finally allow marketers to probe the consumers' brains in order to gain valuable insights on the subconscious processes explaining why a message eventually succeeds or fails. They do so by removing the biggest issue facing conventional advertising research, which is to trust that people have both the will and the capacity to report how they are affected by a specific piece of advertising.

While the field of neuroscience has grown dramatically in the last decade, it has not yet fully penetrated the dark and reclusive hallways of advertising research academia. Why? First, very few marketing researchers have formal training in cognitive neuroscience. Second and more importantly, marketing researchers have long feared the public outcry against potential ethical and privacy issues introduced by the use of neuroimaging technology for commercial purposes. As a result, few scientific neuromarketing studies on advertising effectiveness have yet been published. However, the situation is changing quickly.

Indeed, neuromarketing is fast becoming mainstream. Today, tracking the popularity of the word "neuromarketing" on Google shows a phenomenal progression from just a few hits in 2002 to thousands in 2010. Meanwhile, advertising agencies are beginning to clearly understand the importance of predicting the effectiveness of campaigns by using brain-based tools such as eye tracking, EEG, or fMRI. Finally, the recent weakened economy continues to put pressure on executives to predict and measure the return on the massive dollars they invest in advertising campaigns of all forms. Taking all these factors into account demonstrates that the need for innovative advertising research using the latest discoveries on the brain is both strong and timely.

\section{Measuring Brain Response to Advertising Messages}

There are many ways to measure physiological responses to advertising but there are only three well established noninvasive methods for measuring and mapping brain activity: electroencephalography (EEG), magnetoencephalography (MEG) and functional magnetic resonance imaging ( $\mathrm{ARR}$ ). All three imaging techniques are non-invasive and therefore can be used safely for marketing research purposes. That is why they constitute the bulk of studies that have been published in the last five years. Each method has its pros and cons.

EEG is a rather old technology in neurology but is still considered a good way to measure brain activity. The cells responsible for the biological basis of our cognitive responses are called neurons. We have over 100 billion neurons and trillions of synaptic connections which represent the basis of neural circuitry. In the presence of a particular stimulus like a piece of advertising, neurons fire and produce a tiny electrical current that can be amplified. These electrical currents have multiple patterns of frequencies called brainwaves which are associated with different states of arousal. When EEG is used for a marketing research experiment, electrodes are placed on the scalp of a test subject, typically by using a helmet or a band. Brainwaves can be recorded at very small time intervals. Some of the new EEG bands can record up to 10,000 times per second. This is valuable considering the speed at which we acquire information through our senses and the speed of our thoughts. The limitation of EEG however is that it does not have good spatial resolution which means it cannot precisely locate where the neurons are firing in the brain, especially in deeper, older structures. This is simply because the electrodes on the scalp cannot pick up electrical signals that reside much beyond the cortex. Lastly, since it is estimated that nearly $80 \%$ of our brain activity is used to sustain a critical state called "rest time" or "the default mode" or simply "baseline", it is hardly possible to claim that the brainwaves generated by specific advertising stimuli are entirely produced by the stimuli.

The first psychological studies done using EEG date as far as 1979. Davidson was one of the first cognitive scientists to propose a framework for linking affect and electrical patterns in the brain. His studies and others later validated that electrical patterns were lateralized in the frontal region of the brain. Generally, the measure of alpha-band waves $(8-13 \mathrm{~Hz})$ in the left frontal lobe indicates positive emotions. It is further speculated that such activity is a good predictor of how motivated we are to act. On the other hand, electrical activity in the right frontal lobe is typically correlated with negative emotions. Such emotions generally prepare us to withdraw from an experience.

Though the relative low cost of using EEG has made the technology very popular among neuromarketing agencies in the last 5 years, it is widely considered by cognitive scientists as weak if not dubious for the purpose of understanding and predicting the effects of advertising. While insights gained by using EEG can be helpful to assess the value of a piece of advertising, they are insufficient to help us understand the cognitive process responsible for triggering activity in the entire brain. 
Considered a cousin to EEG, MEG emerged in the midsixties and has gained considerable attention in the last decade because of the tremendous improvements made in measuring and imaging magnetic fields in the brain. As we discussed earlier, brain activity is a function of electrochemical signals between neurons. Neuronal activity creates a magnetic field that can be amplified and mapped by MEG. MEG has excellent temporal resolution, but more importantly, a better spatial resolution than EEG. However, like EEG, MEG is somewhat limited to picking up activity at the surface of the brain; hence it is not a good method for imaging subcortical areas. While the technology is very expensive and has limitations, a few valuable studies have demonstrated that specific frequency bands correlate to controllable cognitive tasks such as recognizing objects, accessing verbal working memory, and recalling specific events. This in fact suggests that the best way to use MEG is to measure activity in areas known or expected to produce activity given specific tasks rather than to conduct exploratory experiments.

So, while MEG is continuing to improve and provides an excellent way to record nearly real-time responses to cognitive events, it is not ideal to conduct marketing research studies investigating both higher cognitive functions (cortical) and emotional (subcortical). Most researchers working with MEG combine both MEG and $f$ MRI in order to optimize both temporal and spatial resolution issues and/or provide the added value of time stamping critical cognitive sequences at the incredible speed of just a few milliseconds.

Unlike both EEG and MEG, the $\mathbf{f M R I}$ modality is based on using an MRI scanner to image the change of blood flow in the brain. When neurons fire, they need to use energy which is transported by the blood flow and quickly metabolized. The key element for a marketing researcher to understand is the contrast of the BOLD signal measured by the fMRI. BOLD is an acronym for Blood Oxygen Level Dependant. When faced with a particular stimulus such as an ad, areas of a subject's brain receive more oxygenated blood flow than they do at rest time. This change creates distortions in the magnetic field emitted by hydrogen protons in the water molecules of our blood. The basis of all $f$ MRI studies is to consider that the change in the BOLD signal is an accurate measure of neuronal activity, even though it does not directly measure electrochemical signals generated by our neurons. While the spatial resolution of fMRI is 10 times better than EEG by providing researchers the ability to image the activity of a voxel (Volume-Pixel), a cube of neurons $(1 \mathrm{~mm} \times 1 \mathrm{~mm} \times 1 \mathrm{~mm}$ in size $)$, the temporal resolution of the technology is considered rather slow. Indeed, there is a delay between the times a neurons fires and the time it takes for the BOLD signal to change: usually a couple of seconds. Nevertheless, $f$ MRI has the major advantage of being able to image deep brain structures, especially those involved in emotional responses. fMRI scanners are also quite expensive but more widely available than MEG equipment. All these factors combined explain why $f$ MRI is the most frequently used brain imaging techniques in the world today and in most likelihood will become the preferred option for neuromarketing scientists for years to come.

\section{What Can We All Learn from Neuromarketing?}

If neuroscience is considered to be in its infancy, neuromarketing is clearly at an embryonic stage. Marketers are just awakening to the possibilities offered by unveiling the brain circuits involved in seeking, choosing, and buying a product. While many of the studies done by neuromarketers are commercial and as such don't go through the standards and review process imposed by academics, enough evidence has been already published to highlight a few core neurocognitive principles at play when consumers perceive advertising messages. Back in 2002, I co-authored the first book on neuromarketing outlining such principles without having the benefit of validating many of my assumptions. Since then however, I have collected tremendous scientific and empirical evidence to support the basis of a sound neuromarketing model. It looks like this:

The brain is responsible for all our consumer behaviors. To function properly, it needs to use a lot of energy. Even though the brain is only $2 \%$ of our body mass, it burns nearly $20 \%$ of our energy. Most of the functions we need to go through a day are managed by the brain below our level of consciousness. This explains why nearly $80 \%$ of our brain energy is necessary to sustain our rest state or default mode, a critical aspect of brain functioning which continues to puzzle neuroscientists. Clearly, we only use about $20 \%$ of our brain consciously. Worse, we do not control the bulk of our attention since we are too busy scanning the environment for potential threats. Because nothing matters more than survival, we are in fact largely controlled by the most ancient part of our brain known as the R-complex or the reptilian brain.

The reptilian brain has developed over millions of years. It is pre-verbal, does not understand complex messages, and seeks pain avoidance over thrills. It is the part of the brain that makes us extremely selfish and drives our strong preference for mental shortcuts over long deliberations. The most powerful aspect of the reptilian brain is the fact that it is able to process visual stimuli without the use of the visual cortex. This is why we prefer images over words and experiences over explanations. Antonio Damasio, a wellknown neuroscientist and respected author once said, "We are not thinking machines that feel, we are feeling machines 
that think". What Damasio and many others have demonstrated is that while we appreciate and even worship our cognitive abilities, the brain has been dependent on instinctual responses for millions of years. And it will continue to do so for a long time since biological adaptation to a fast changing environment is too slow. What does this mean from a neuromarketing perspective? It means that there are specific principles that should apply to advertising messages in order to optimize the processing of information at the level of our brain. In today's world, we receive an average of 10,000 messages per day. This volume of data is largely irrelevant unless it speaks directly to the reptilian brain.

Back at the lab, John feels better. The scan is done. This was not so hard, he thinks. Plus he took advantage of the opportunity to insert a picture of his family at the end of the experiment. The neuroscientist in charge of the study told him that he would love to see his brain at the precise moment he would feel love and attachment. Wow!

Neuromarketing is here to stay. And it will evolve, like humans - and even brands - do. Consumers like you may never see the difference in the messages that are refined or produced as a result of gaining a better understanding of our buying decision process. Ethical issues will continue to surface but standards have already been adopted to make sure that neuromarketing research is conducted with respect and transparency. Let's also remember that many advertising messages are not commercial either. Countless campaigns aim at changing people's self-destructive behaviors. For example, there is a tremendous need to improve our ability to convince people not to smoke or not text and drive. Words don't work. Pictures do. Why? It is a reptilian brain thing!

\section{Further Reading}

Ariely, D., \& Berns, G. S. 2010. Neuromarketing: The hope and hype of neuroimaging in business. Nature reviews Neuroscience (March).

Fugate, D. L. 2007. Neuromarketing: A layman's look at neuroscience and its potential application to marketing practice. Journal of Consumer Marketing, 24(7), 385-394.

Glimcher, P. W. 2009. Neuroeconomics: Decision-making and the brain. London: Elsevier.

Kenning, P., Plassmann, H., \& Ahlert, D. 2007. Applications of functional magnetic resonance imaging for market research. Qualitative Market Research, 2, 135-152.

Knutson, B., Rick, S., Wimmer, E. G., Prelec, D., \& Loewenstein, G. 2007. Neural predictors of purchases. Neuron, 53, 147-156.

Lee, N., Broderick, L., \& Chamberlain, L. 2006. What is "neuromarketing'? A discussion and agenda for future research. International Journal of Psychophysiology, 63, 200-204.

Christophe Morin received his MBA in Marketing Research and Organizational Behavior from Bowling Green State University and is currently pursuing a $\mathrm{PhD}$ in Media Psychology with Fielding Graduate School in Santa Barbara, California. Before co-founding SalesBrain in 2002, he held several key executive positions in technology, retail, and leadership companies. Since 2002, he has lectured extensively on the subject of neuromarketing in the US, in Europe and Asia delivering over 500 workshops on the subject. He is co-author of Neuromarketing: Understanding the Buy Buttons in Your Customer's Brain (Nelson). 